\title{
Periodic fever, aphthous stomatitis, pharyngitis and cervical adenitis syndrome persisting to adulthood - an example of a diagnostic and therapeutic challenge
}

\author{
Ewa Więsik-Szewczyk ${ }^{1}$, Beata Wolska-Kuśnierz², Karina Jahnz-Różyk ${ }^{1}$ \\ ${ }^{1}$ Department of Internal Medicine, Pneumonology, Allergology and Clinical Immunology, Central Clinical Hospital \\ of the Ministry of National Defense, Military Institute of Medicine, Warsaw, Poland \\ ${ }^{2}$ Department of Immunology, Children's Memorial Health Institute, Warsaw, Poland
}

\begin{abstract}
Periodic fever, aphthous stomatitis, pharyngitis and cervical adenitis (PFAPA) syndrome is the most common cause of periodic fever in childhood. Reports of adult patients are sparse. In adults the clinical picture is more heterogeneous than in children, so PFAPA can be a real diagnostic challenge. Data regarding treatment efficacy and disease outcome are available mainly for children, whereas for adult patients they are limited and conflicting. Our aim is to increase the awareness about PFAPA among clinical practitioners. We present a case of PFAPA beginning in childhood and without resolution of symptoms in maturity. In our case the diagnostic delay was 15 years. We treated the patient with a prophylactic dose of colchicine. Colchicine helped to control flares and significantly improved the patient's quality of life. Periodic fever, aphthous stomatitis, pharyngitis and cervical adenitis in adults is a rare disease, but it should be included in the differential diagnosis of fever of unknown origin in adults.
\end{abstract}

Key words: polygenic autoinflammatory diseases, adults, periodic fever, aphthous stomatitis, pharyngitis, cervical adenitis, colchicine, treatment.

\section{Introduction}

Periodic fever, aphthous stomatitis, pharyngitis and cervical adenitis (PFAPA) syndrome is the most common cause of self-limiting periodic fever in childhood. In the long term, spontaneous resolution of symptoms before adolescence has been treated as a rule.

Descriptions in adults are rare, but the possibility of diagnosis of the disease in adults should not be excluded, including two scenarios - adult onset - PFAPA and PFAPA persisting from childhood until adulthood [1-4]. In adults a proper diagnosis can be delayed, leading to unnecessary fear of unpredictable, mysterious diseases and diminished quality of life.

\section{Case report}

A 32-year-old woman was transferred to our center from a pediatric Immunology Department for further care. From the age of two she suffered from attacks of fever which recurred irregularly every 1-2 months, then they became more regular every 4-5 weeks and lasted 3-5 days from puberty. Fevers accompanied aphthae in the mouth, exudative pharyngitis, cervical lymph node enlargement, abdominal pain and headaches. The patient's family history was unremarkable. In laboratory investigations mild leukocytosis, erythrocyte sedimentation rate (ESR) and C-reactive protein (CRP) elevation were documented during attacks. Serum amyloid A (SAA) was not available this time. Between the age of 2 to 17 she was hospitalized

Address for correspondence:

Ewa Więsik-Szewczyk, Department of Internal Medicine, Pneumonology, Allergology and Clinical Immunology, Central Clinical Hospital of the Ministry of National Defense, Military Institute of Medicine, 128 Szaserów St., 04-141 Warsaw, Poland, e-mail: ewa.w.szewczyk@gmail.com

Submitted: 08.07.2019; Accepted: 25.09.2019 
35 times without relevant conclusions. Lymphoma, systemic lupus erythematosus, vasculitis and systemic juvenile idiopathic arthritis were excluded. Despite negative bacterial culture each time a course of antibiotics was ordered. At the age of 10, she had adenotomy without any impact on the disease course.

At the age of 17 she was referred to a clinical immunologist due to an elevated level of immunoglobulin D. The autoinflammatory syndrome was suspected. The patient received $1 \mathrm{mg} / \mathrm{kg} /$ day prednisolone at the onset of the episode with improvement, but not prevention of attacks. PFAPA syndrome was diagnosed. Symptomatic treatment with prednisone $0.5 \mathrm{mg} / \mathrm{kg}$ to abate the attacks was introduced. On further follow-up, as symptoms recurred, from the age of 30, colchicine $1 \mathrm{mg} /$ day was introduced for prophylaxis. Less and milder fever attacks were observed, with good patient's compliance. Controlled CRP and SAA were within the reference range. Genetic tests for mutations related to monogenic hereditary autoinflammatory syndromes: MVK (exons 8 and 10) for mevalonate kinase deficiency (MKD), TNFRSF1A (exons 2, 3 and 4) for tumor necrosis factor receptor-associated periodic syndrome (TRAPS), and MEFV (exons 1, 2, 3, 5, 9 and 10) for familial Mediterranean fever (FMF) were negative.

At the age of 29 our patient was diagnosed with Hashimoto thyroiditis and at the age of 32 with Graves' disease. An endocrinologist ordered thiamazole and asked for the termination of colchicine. After 2 months without colchicine aphthous inflammation and low grade fever episodes with CRP elevations recurred. Colchicine was safely reintroduced with a good effect in the further 5-year follow-up. At the age of 37 the patient was also diagnosed with hypoglycemia and insulin resistance.

\section{Discussion}

Our patient had PFAPA symptoms from early childhood but physicians had misinterpreted them for many years. The diagnostic delay was 15 years, leading to unnecessary hospitalizations. PFAPA syndrome was first described in 1987 by Marshall et al. [5] and is the most common cause of periodic fever in children. Table I presents the assumptions of the first PFAPA criteria based on Marshal observations.

The syndrome is characterized by fever (generally $>39^{\circ} \mathrm{C}$ ) lasting from 3 to 6 days, with recurrence every 3-8 weeks accompanied by at least one of the cardinal symptoms: aphthous stomatitis, pharyngitis, and cervical adenitis [6]. Headache, abdominal pain, nausea, vomiting, chills, malaise, arthralgia and myalgia can coexist or even dominate [7]. Patients are asymptomatic between episodes, and show normal growth and development. The diagnosis is based on clinical history, physical findings and exclusion of other causes of fever.

\section{Differential diagnosis}

The main differential diagnosis of PFAPA depends on the patient's age, but the diagnosis is much easier to confirm in childhood. Initial differential work-up includes infectious pharyngitis and cyclic neutropenia (CN). The absence of symptoms typical for upper respiratory tract infection, no seasonal pattern of flares, and negative pharyngeal swabs are helpful. Often parents report that antibiotics did not improve or change the child's condition, in contrast with one dose of corticoids, which given at the onset of symptoms can halt the flare. A rapid response to a single dose of corticosteroids confirms the diagnosis [8].

In CN fever usually occurs every 18-24 days and is accompanied by an absolute decrease in neutrophil count below 200/ $\mu$ l on at least 3-5 consecutive days per cycle of each of three regularly spaced cycles. Fever in $\mathrm{CN}$ does not respond to corticosteroids. Serial blood cell count every week for 6 weeks is recommended to rule out CN.

PFAPA generally is self-limiting by adolescent age. In the literature there are descriptions of persistence of the syndrome into adulthood and also adult onset of PFAPA. The evidence suggests that the criterion "age onset less than 5 years" should not be always considered [8] (Table II).

Table I. Diagnostic criteria of PFAPA based on Marshal [5]

\begin{tabular}{|l|}
\hline 1. Regularly recurring fevers with early age of onset (patients $<5$ years of age) \\
\hline 2. Constitutional symptoms in the absence of upper respiratory infection with at least 1 of the following clinical signs: \\
\hline aphthous stomatitis \\
\hline pharyngitis \\
\hline cervical lymphadenitis \\
\hline 4. Cxclusion of cyclic neutropenia \\
\hline 5. Normal growth and development without long-term sequelae of the disease \\
\hline
\end{tabular}


Table II. Diagnostic criteria of PFAPA according to Padeh [8]

\begin{tabular}{|l|}
\hline 1. Monthly fevers - cyclic fever at any age group \\
\hline 2. Exudative tonsillitis + negative throat culture \\
\hline 3. Cervical lymphadenitis \\
\hline 4. Possibly aphthous stomatitis \\
\hline 5. Completely asymptomatic interval between episodes \\
\hline $\begin{array}{l}\text { 6. Rapid response to a single dose of corticosteroids } \\
\text { (60 mg prednisone) }\end{array}$ \\
\hline
\end{tabular}

The problem is that not all adult patients present the full clinical picture of PFAPA. In a series of 36 adult patients collected from the scientific medical literature 3 cardinal signs occurred in less than $50 \%$ of cases [9]. In comparison with children, adults complained significantly more often of arthralgia and myalgia, while chills and aphthae occurred less [10]. Ten of 15 adult PFAPA patients had 2 or 3 symptom-free intervals of 2-6 months during the course of disease. It is suspected that in adults the course of disease may often be interspersed with symptom-free periods of some duration [11]. The comparison between unrelated 85 children and 30 adults with PFAPA revealed that the flares were significantly more frequent in children, but attack duration was longer in adults. Clockwork periodicity of fever and recurrent pharyngitis were more common in the pediatric population [12]. In adults also a relapse after years of remission is possible [13].

Cantarini et al. [14] proposed a simple diagnostic criteria to identify the adult onset of PFAPA (aged >16) among patients with fever of unknown origin (FUO) which include: recurrent fever accompanied by erythematous pharyngitis and/or cervical lymphadenitis, and increased inflammatory markers during attacks and a symptom-free interval (Table III).

These criteria should be applied if infective, autoimmune, and neoplastic diseases as well as monogenic autoinflammatory diseases (AIDs) and febrile polygenic AIDs were excluded. In addition, throat swab performed during fever has to be negative and antibiotics ineffective [14].

In adults, with the atypical clinical picture, the probability of monogenic, hereditary AID should be assessed. However, in comparison to PFAPA, they belong to the group of ultra-rare diseases. The most common monogenic AIDs are familial Mediterranean fever (FMF), NLRP3 - associated autoinflammatory diseases also known as cryopyrin-associated autoinflammatory syndrome (NLRP3-AIDS, CAPS), tumor necrosis factor receptor-associated periodic fever syndrome (TRAPS) and mevalonate kinase deficiency (MKD, formerly known as hyperimmunoglobulin D syndrome - HIDS) [15].

In our case during follow-up symptoms did not resolve with age, although they became less regular, which made the diagnosis even more difficult. We decided to perform genetic testing for 3 common monogenic AIDs. All results were negative. However it is not a standard approach. The criteria for qualification of adult patients with PFAPA suspicion for genetic testing in clinical practice are not firmly established. Some authors favor the opinion that the final diagnosis of adult PFAPA should be made only after the exclusion of monogenic AIDs, while others think that it is not necessary. Genetic studies have shown that heterozygous variants of MEFV are also found in PFAPA and healthy population, and do not support diagnosis of FMF and do not change the management $[16,17]$. The pathogenic role and phenotypegenotype correlation in PFAPA is a matter of debate [18].

In our opinion the decision has to be made in a caseby-case analysis in tertiary reference centers experienced with management of AIDs.

\section{Autoimmunity and autoinflammation}

The next unresolved issue is coexistence of PFAPA syndrome and autoimmune diseases. An inflammatory response in PFAPA is also driven by a Th1-type adaptive immune response, which also dominates in several autoimmune diseases, for example, Hashimoto's thyroiditis, Graves' disease, Crohn's disease, psoriasis, type 1 diabetes, IgA nephropathy and rheumatoid arthritis [19-21].

Table III. Proposed diagnostic criteria for adult-onset PFAPA according to Cantarini [14]

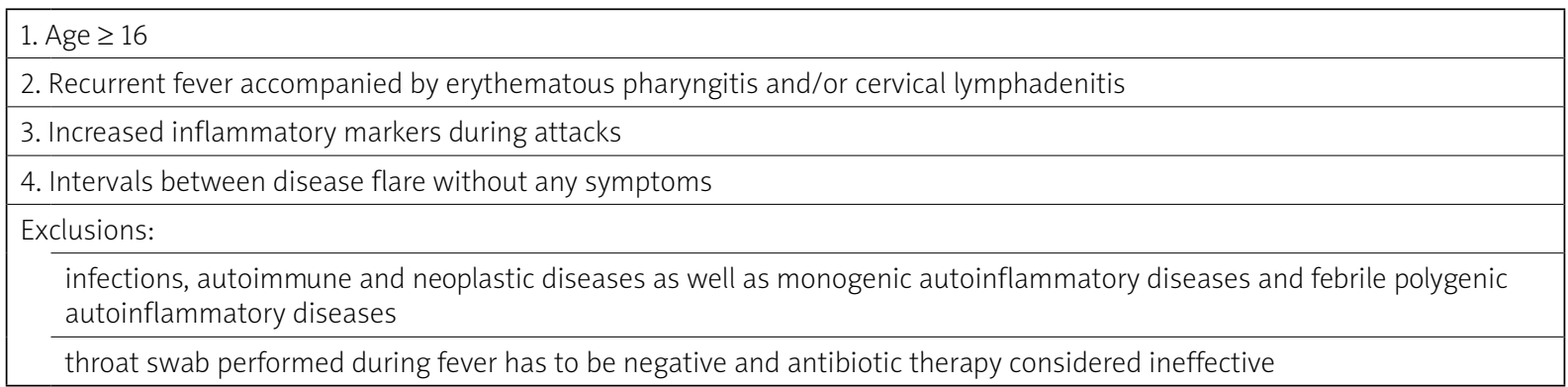


In our case both Hashimoto's thyroiditis and Graves' disease and hypoglycemia were diagnosed on follow-up. Further studies are necessary to show whether it is a coincidence or common pathogenic pattern.

\section{Management}

The current PFAPA pharmacological management includes corticosteroids to resolve flares, colchicine for prophylaxis of attacks, and interleukin 1 (IL-1) inhibitors in severe, refractory cases. Tonsillectomy is an option in selected cases. However, data regarding efficacy of treatment in adult patients are limited and conflicting [22]. The use of prednisone at the onset of fever in pediatric patients with PFAPA proved to be an effective strategy. In some countries steroids are used during each episode, in others to postpone the attack that occurs at an inconvenient time [23]. In adults prednisone administered at a dose of 50-60 mg/day in 33/36 patients gave a complete response in 28, partial in 4 and no response in 1 [9].

The safety of a single steroid dose administration is widely accepted and side effects are rare. However, the steroid application may shorten the interval between episodes. In our case steroids on demand were stopped after 12 years. Colchicine $1 \mathrm{mg} /$ day was well tolerated and effective in prophylaxis of attacks, although it had to be given systematically as after a 10-week pause symptoms reappeared. Data published mainly from pediatric populations suggest that colchicine may be a treatment option [24-26]. Colchicine significantly prolonged the interval between episodes [24, 26]. In a 6-month open label, randomized, controlled study of 18 patients aged 4-11 years colchicine reduced the number of attacks [25]. Colchicine may be more effective in patients with less completed PFAPA phenotype and MEV heterozygosity [26, 27]. From our experience it seems to be safe and it is at least a partially effective option. The open question is how long treatment has to be continued.

In 2 small non-blinded trials tonsillectomy with or without adenoidectomy gave a clinical benefit in children [28]. Unfortunately available data in adults suggest that tonsillectomy is not effective. Of 36 reported adults patients 16 underwent surgery with a complete response in only 1 case [9].

In the literature there are case reports of adult patients with refractory PFAPA treated with the IL-1 inhibitors anakinra [29] and canakinumab [30]. The IL-1 inhibition due to the pathogenesis of the disease is a very tempting option but the cost effectiveness of therapy is the main concern.

\section{Conclusions}

The presented case can improve the knowledge of PFAPA syndrome and underscore the possibility of diag- nostic delay of this typically pediatric disease to adulthood or adult onset of PFAPA. Although we support the use of colchicine as prophylactic treatment, its therapeutic effect in PFAPA syndrome is undetermined and has to be validated in future prospective studies. Although PFAPA is a disease with a favorable prognosis, it can limit patients' everyday activity and quality of life. We also recommend the follow-up of patients to pay attention to coexistence of Th1 mediated autoimmune diseases.

The authors declare no conflict of interest.

\section{References}

1. Hernández-Rodriguez J, Ruiz-Ortiz E, Tomé A, et al. Clinical and genetic characterization of the autoinflammatory diseases diagnosed in an adult reference center. Autoimmun Rev 2016; 15: 9-15.

2. Cantarini L, Rigante D. Adult-onset autoinflammatory disorders: a still debated entity? Clin Exp Rheumatol 2015; 33: 137-140.

3. Marques DP, Rocha S, Manso M, Domingos R. Periodic Fever with Pharyngitis, Aphthous Stomatitis and Cervical Adenitis Syndrome: A rare cause of fever in adults. Eur J Case Rep Intern Med 2019; 6: 001041.

4. Alam F, Hammoudeh M. An underlooked cause of periodic fever (PFAPA) in an adult patient with no response to tonsillectomy. Case Rep Rheumatol 2018; 2018: 6580835.

5. Marshall GS, Edwards KM, Butler J, Lawton AR. Syndrome of periodic fever, pharyngitis, and aphthous stomatitis. J Pediatr 1987; 110: 43-46.

6. Thomas KT, Feder Jr HM, Lawton AR, Edwards KM. Periodic fever syndrome in children. J Pediatr 1999; 135: 15-21.

7. Tasher D, Somekh E, Dalal I. PFAPA syndrome: new clinical aspects disclosed. Arch Dis Child 2006; 91: 981-986.

8. Padeh S, Brezniak N, Zemer D, et al. Periodic fever, aphthous stomatitis, pharyngitis, and adenopathy syndrome: clinical characteristics and outcome. J Pediatr 1999; 135: 98-101.

9. Cattalini M, Soliani M, Rigante D, et al. Basic characteristic of adults with periodic fever, aphthous stomatitis, pharyngitis, and adenopathy syndrome in comparison with typical pediatric expression of disease. Mediators Inflamm 2015; 2015: 570418.

10. Padeh S, Stoffman N, Berkun Y. Periodic fever accompanied by aphthous stomatitis, pharyngitis and cervical adenitis syndrome (PFAPA syndrome) in adults. Isr Med Assoc J 2008; 10 : 358-360.

11. Kutsuna S, Ohmagari N, Tanizaki R, et al. The first case of adult-onset PFAPA syndrome in Japan. Modern Rheumatol 2016; 26: 286-287.

12. Rigante D, Vitale A, Natale MF, et al. A comprehensive comparison between pediatric and adult patients with periodic fever, aphthous stomatitis, pharyngitis, and cervical adenopathy (PFAPA) syndrome. Clin Rheumatol 2017; 36: 463-468.

13. Vitale A, Orlando I, Lopaco G, et al. Demographic, clinical and therapeutic findings in a monocentric cohort of adult patients with suspected PFAPA syndrome. Clin Exp Rheumatol 2016; 34 (6 Suppl 102): 77-81. 
14. Cantarini L, Vitale A, Sicignano LL, et al. Diagnostic Criteria for Adult-Onset Periodic Fever, Aphthous Stomatitis, Pharyngitis, and Cervical Adenitis (PFAPA) Syndrome. Front Immunol 2017; 8: 1018.

15. Gattorno M, Hofer M, Federici S, et al. Classification criteria for autoinflammatory recurrent fevers. Ann Rheum Dis 2019; 78 1025-1032.

16. Cazeneuve C, Geneviève D, Amselem S, et al. MEFV gene analysis in PFAPA. J Pediatr 2003; 143: 140-141.

17. Taniuchi S, Nishikomori R, Iharada A, et al. MEFV Variants in Patients with PFAPA Syndrome in Japan. Open Rheumatology J 2013; 7: 22-25.

18. Cantarini L, Rigante D, Merlini G, et al. The expanding spectrum of low-penetrance TNFRSF1A gene variants in adults presenting with recurrent inflammatory attacks: clinical manifestations and long-term follow-up. Semin Arthritis Rheum 2014 43: 818-823.

19. Kraszewska-Głomba B, Matkowska-Kocjan A, Szenborn L. The Pathogenesis of Periodic Fever, Aphthous Stomatitis, Pharyngitis, and Cervical Adenitis Syndrome: A Review of Current Research. Mediators Inflamm 2015; 2015: 563876.

20. Cazzato M, Neri R, Possemato N, et al. A case of adult periodic fever, aphthous stomatitis, pharyngitis, and cervical adenitis (PFAPA) syndrome associated with endocapillary proliferative glomerulonephritis. Clin Rheumatol 2013; 32 (Suppl 1): S33-36.

21. Sugimoto K, Fujita S, Miyazawa T, et al. Periodic fever, aphthous stomatitis, pharyngitis, and adenitis (PFAPA) syndrome and IgA nephropathy. Pediatr Nephrol 2013; 28: 151-154.

22. Gaggiano C, Rigante D, Sota J, et al. Treatment options for periodic fever, aphthous stomatitis, pharyngitis, and cervical adenitis (PFAPA) syndrome in children and adults: a narrative review. Clin Rheumatol 2019; 38: 11-17.
23. Wekell P, Karlsson A, Berg S, Fasth A. Review of autoinflammatory diseases, with a special focus on periodic fever, aphthous stomatitis, pharyngitis and cervical adenitis syndrome. Acta Paediatr 2016; 105: 1140-1151.

24. Tasher D, Stein M, Dalal I, Somekh E. Colchicine prophylaxis for frequent periodic fever, aphthous stomatitis, pharyngitis and adenitis episodes. Acta Paediatr 2008; 97: 1090-1092.

25. Butbul Aviel Y, Tatour S, Gershoni Baruch R, Brik R. Colchicine as therapeutic option in periodic fever, aphthous stomatitis, pharyngitis, cervical adenitis (PFAPA) syndrome. Semin Arthritis Rheum 2016; 45: 471-474.

26. Gunes M, Cekic S, Kilic SS. Is colchicine more effective to prevent PFAPA episodes in MEFV variants? Pediatr Int 2017; 59: 655-660.

27. Dusser P, Hentgen V, Neven B, Koné-Paut I. Is colchicine an effective treatment in periodic fever, aphtous stomatitis, pharyngitis, cervical adenitis (PFAPA) syndrome? Joint Bone Spine 2016; 86: 406-411.

28. Burton MJ, Pollard AJ, Ramsden JD, et al. Tonsillectomy for periodic fever, aphthous stomatitis, pharyngitis and cervical adenitis syndrome (PFAPA). Cochrane Database Syst Rev 2014; 9: CD008669.

29. Cantarini L, Vitale M, Galeazzi M, Frediani B. A case of resistant adult-onset periodic fever, aphtous stomatitis, pharyngitis and cervical adenitis (PFAPA) syndrome responsive to anakinra. Clin Exp Rhumatol 2012; 30: 593.

30. Lopalco G, Rigante D, Vitale A, et al. Canakinumab efficacy in refractory adult-onset PFAPA syndrome. Int J Rheum Dis 2015; 20: 1050-1051. 